\title{
Frontières
}

\section{Le lien entre les concepts de guerre et de martyre au début de l'islam}

\section{Ali G. Dizboni}

Volume 15, numéro 2, printemps 2003

Guerre, mort amère

URI : https://id.erudit.org/iderudit/1073813ar

DOI : https://doi.org/10.7202/1073813ar

Aller au sommaire du numéro

Éditeur(s)

Université du Québec à Montréal

ISSN

1180-3479 (imprimé)

1916-0976 (numérique)

Découvrir la revue

Citer cet article

Dizboni, A. G. (2003). Le lien entre les concepts de guerre et de martyre au début de l'islam. Frontières, 15(2), 12-16. https://doi.org/10.7202/1073813ar
Résumé de l'article

Cet article explore le lien entre les deux concepts de guerre et de martyre et leur statut au début de l'islam dans le Coran ainsi que dans les paroles de Mahomet et de ses compagnons. Afin de montrer l'interaction entre le texte coranique et son contexte arabe, nous ouvrons la discussion par un débat sur la culture de guerre pendant la période préislamique. Ceci nous permet d'observer une certaine continuité qui, avec des justifications normatives différentes, demeurera présente au niveau de la valorisation de la guerre et du sacrifice physique. 


\section{Résumé}

Cet article explore le lien entre les deux concepts de guerre et de martyre et leur statut au début de l'islam dans le Coran ainsi que dans les paroles de Mahomet et de ses compagnons. Afin de montrer l'interaction entre le texte coranique et son contexte arabe, nous ouvrons la discussion par un débat sur la culture de guerre pendant la période préislamique. Ceci nous permet d'observer une certaine continuité qui, avec des justifications normatives différentes, demeurera présente au niveau de la valorisation de la guerre et du sacrifice physique.

Mots clés : islam - martyre-djihadCoran-hadith.

\section{Abstract \\ This article explores the relation between two concepts of war and martyr and their status in the early Islam both in Koran's text and in Mahomet and his followers' statements. To show the interaction be- tween Koran and its Arab context, the paper starts by discussing the pre-Islamic attitudes towards war. This discussion allows us to observe some continuity between two periods. With divergent normative justifications, war and human sacrifices remain valued.}

Key words: Islam - Martyr - Jihad Koran-Hadith.

\section{LE LIEN ENTRE LES CONCEPTS DE GUERRE ET DE MARTYRE AU DÉBUT DE L'ISLAM}

\author{
Ali G. Dizboni, Ph.D., \\ professeur adjoint \\ Collège militaire du Canada (Kingston).
}

Si on pouvait appeler le siècle précédent celui des idéologies laïques, le troisième millénaire débute, certes, comme l'avait prédit André Malraux et affirmé Samuel Huntington (2000), avec un retour de la religion. L'islamisme (ou islam politique) est la figure dominante de cette grande rentrée millénaire. Le point culminant (jusqu'à présent) dans l'enchaînement des actions islamistes depuis les années 1980, a été, faut-il le rappeler, le 11 septembre 2001. Les «fous d'Allah» ne sont désormais plus les associés désirés contre le mal soviétique mais sont devenus l'incarnation de l'axe de Mal.

Depuis l'attentat terroriste du 11 septembre 2001, la curiosité occidentale traite l'Orient islamique tantôt comme "la demeure du mal », tantôt comme "l'autre » qui est à la fois proche et lointain. Pour qu'elle soit fructueuse, la curiosité doit dépasser le simple intérêt folklorique et devenir scientifique et globale sans toutefois tomber dans le relativisme. C'est dans ce cadre que l'article s'adresse à cette curiosité intellectuelle en discutant de l'un des concepts les plus médiatisés, celui de martyre, tel qu'il a été défini lors de la genèse de l'islam.
Quelles sont les caractéristiques principales du martyre dans l'islam classique? Pour répondre à cette question, notre réflexion s'articulera autour de trois éléments. Premièrement, on voudra reconnaître le rôle de la guerre et du sacrifice dans la culture arabe préislamique $\left(\mathrm{VI}^{\mathrm{e}}\right.$ siècle après J.-C.). Lutter et se sacrifier au nom de l'honneur tribal est un élément important à souligner, et en comprendre les raisons nous aidera à situer le discours coranique de martyre dans son contexte historique. Deuxièmement, on analysera dans quelle mesure la vision islamique de la guerre (djihad) s'inscrit dans la continuité ou représente une rupture par rapport aux guerres tribales préislamiques. Troisièmement, on parlera du rapport entre les concepts de martyre et de djihad pour mieux comprendre le statut du martyre en islam

Notre démarche vise avant tout à présenter le concept de martyre aux débuts de l'islam ; il ne se veut pas une discussion des conditions qui autorisent la guerre sainte (défensive ou offensive). Notre présentation porte plutôt exclusivement sur l'importance du djihad (quitte à savoir si elle était défensive ou offensive). La méthode de notre analyse sera discursive et interprétative se basant sur des textes de poésie, sur le Coran et sur les hadiths (les actes et les paroles de Mahomet recueillis en textes). 


\section{LA PÉRIODE PRÉISLAMIQUE ET LA GUERRE}

Le Coran qualifie la période qui précède les révélations de Dieu à Mahomet en 610 par le terme de jahiliyya ou ignorance. Cette appellation s'appliquait essentiellement aux populations de la partie nord (le berceau de l'islam) de la péninsule arabique (en était exclu le Sud, le Yemen contemporain). Majoritairement composées de nomades et de bédouins (Lammens, 1914, p. 185), ces tribus arabes étaient polythéistes et donc «ignorantes » selon le discours du Coran.

La guerre était le modus operandi pour régler les différends entre tribus et au sein des tribus. La valorisation de la guerre atteint le point où elle devient le critère par excellence de la noblesse personnelle et de la vertu morale.

Deux facteurs ont essentiellement façonné cette perception de la guerre : le climat et l'espace géographiques très durs, et l'anarchie politique. $\mathrm{Au}$ début du $\mathrm{XX}^{\mathrm{e}}$ siècle, Lammens (1914, p. 143) insistait déjà sur le fait «[...] que le climat de la Péninsule appartient à la catégorie des climats rigoureux, [fait que] personne ne songe à contester ». Shahid (1970, p. 3) considère l'Arabie (d'avant la découverte du pétrole) comme la victime de natura maligna au point où on appelle sa majeure partie le «désert vide». La géographie a donc joué un rôle important dans la vie des Arabes des débuts de l'islam. Djaït (1989, p. 6) affirme à son tour qu'en Arabie «plus qu'ailleurs, le climat a modelé le genre de vie et donc l'histoire».

$\mathrm{Au}$ contexte naturel, il faut ajouter l'anarchie politique comme facteur d'instabilité et de conflits sanglants. En effet, l'absence de toute autorité centrale supratribale était à la fois cause et effet de conflit. Dans ce Wild East, chaque tribu avait les mains libres dans sa région et la puissance faisait la loi. Lammens (1914, p. 138) parle dans les termes suivants de cette anarchie :

Le siècle antérieur à l'établissement de l'hégire fut pourtant une période d'anarchie politique, de véritable décomposition intérieure [...] Ce fut une époque de luttes fratricides entre les tribus où une course de chevaux, des discussions relatives au droit de pacage suffisaient pour déchaîner d'interminables guerres civiles; où même les cités, comme Médine, ont connu seulement des trêves entre les hostilités.

Les tribus se vantaient de cette liberté, mais l'anarchie créait un besoin de protection chez les bédouins que seule la tribu pouvait offrir. En garantissant une certaine protection physique et l'accès aux ressources d'importance vitale, la tribu devenait aussi le cadre primaire de l'identité. Dans un tel contexte, la défense de l'honneur tribal devient la première cause de conflits sanglants. Ibn Khaldoun, le fameux historien musulman du XII ${ }^{\mathrm{e}}$ siècle, a formulé la notion de asabyya pour décrire ce chauvinisme tribal. Par ailleurs, cet attachement «inconditionnel et sans borne» selon certains observateurs (Hitti, 1968, p. 27) est bien décrit par ce proverbe arabe: "Sois loyal à ta tribu. Son pouvoir sur ses membres est assez grand pour enlever l'épouse à son mari. » Se soumettre à une centralisation politique quelconque, qu'elle soit basée sur un contrat social ou sur un lien politique trans-tribal, devient inacceptable. Comme écrivait Ibn Khaldoun (1868, p. 142) :

Le naturel farouche des Arabes

[Arabes du Nord au VI ${ }^{\mathrm{e}}$ siècle]

en a fait une race de pillards et de

brigands [...] Les habitudes et les usages de la vie nomade ont fait des Arabes un peuple rude et farouche [...] Ajoutons qu'ils négligent tous les soins du gouvernement [...] Menant une vie presque sauvage, ils acquièrent une grossièreté des mœurs, une arrogance, et un esprit de jalousie qui les indisposent contre toute autorité.
Sous l'influence de ces sentiments, ils se soutiennent les uns les autres; ils se prêtent un mutuel secours et se font redouter de leurs ennemis... Pour demeurer ensemble dans l'umran badawi, on doit avoir le moyen de se défendre. L'esprit de corps, c'est le sentiment qui porte à résister, à repousser l'ennemi, à protéger ses amis, à venger ses injures.

Le peuple qui en est dépourvu ne saurait faire rien qui vaille.

Les seules sources qui nous sont parvenues de la période préislamique sont des poèmes de jahili transmis et préservés oralement. Leur thème principal reste l'honneur tribal et la glorification de la guerre. Par ses qualités d'orateur, le poète arabe avait un grand prestige (Milot, 1993, p. 29). Il servait de machine de propagande, voué à la défense de la cause de son clan et contre les adversaires de celui-ci. Par exemple, un des proverbes de cette période les mieux connus dit: «si vous trouvez vos proches en danger vous devez les aider quitte à savoir s'ils ont raison ou tort».

Les poèmes de jahili exaltent à outrance la guerre. La guerre pour la guerre est quelque chose de normal. Dans un poème très connu, Abid (Donner, 1991, p. 61)

\section{LA VALORISATION DE LA GUERRE ATTEINT LE POINT}

OÙ ELLE DEVIENT LE CRITÈRE PAR EXCELLENCE DE LA NOBLESSE PERSONNELLE ET DE LA VERTU MORALE.

Ces facteurs géographiques et politiques ont comme effet cumulatif de créer une situation de guerre quasi permanente. Selon Shahid (1970, p. 5), la guerre devient ainsi une «institution naturelle» où la violence et le chauvinisme tribal se renforcent l'un l'autre. Khaldoun (1868, p. 296) décrit ainsi l'institution:

\section{[...] pour protéger leur campement} contre les ennemis de dehors, chacune [chaque tribu] a une troupe d'élite composée des meilleurs guerriers et de leurs jeunes gens les plus distingués par leur bravoure. Mais cette bande ne serait jamais assez forte pour repousser des attaques, à moins d'appartenir à la même famille et d'avoir pour l'animer le même esprit de corps. Voilà ce qui rend les troupes composées d'Arabes [du désert], (umran badawi), si fortes et si redoutables; chaque combattant n'a qu'une seule pensée, celle de protéger sa tribu et sa famille... décrit ainsi la manie de la guerre: "Oui, fils de guerre que je suis / j'attise continuellement sa flamme et quand elle s'affaiblit je la ramène à son paroxysme $»^{1}$. Ce goût pour la guerre prend parfois le dessus sur la défense absolue de ses proches. Comme le dit Hitti (1968, p. 25) citant un poète arabe : «notre métier est de porter des raids contre notre ennemi / contre notre voisin ou même contre nos frères quand il n'y a personne à attaquer que nos frères ».

\section{LA PÉRIODE ISLAMIQUE ET LE DISCOURS DE LA GUERRE}

L'islam a été décrit comme une révolution de la société arabe (Ghunaimi, 1968, p. 20 ; Djaït, 1989). Cette révolution est bien visible lors de l'établissement de la cité-État de Médine par Mahomet en 613. Grâce à ce projet politico-religieux, les tribus d'Arabie ont été unies, mettant fin à l'anarchie politique de l'époque. Pourtant, comme d'autres révolutions, la venue de l'islam n'a pas provoqué de rupture totale avec la 
culture préislamique. Certains éléments de continuité ont survécu dans la mesure où les mêmes conditions se sont maintenues.

\section{CONTINUITÉ}

La guerre reste un facteur de continuité. Cela est vrai dans la mesure où la guerre est exaltée et glorifiée par la nouvelle foi. Par contre, elle ne sera pas faite au nom de sa tribu mais plutôt dans la perspective d'un État islamique, celui de Médine, en voie de consolidation, puis en voie d'expansion (djihad). Certes, les règles, les objectifs et les formes de la guerre ont changé ; mais au moins au niveau de la valorisation de la guerre, il n'y a pas de rupture totale avec la tradition guerrière préislamique. $\mathrm{Kab} i b n$ Malik, un des compagnons très connus de Mahomet, souligne la continuité des mœurs :

Nos ancêtres nous ont appris comment attaquer

nous apprendrons à nos enfants

l'habileté guerrière des héros

et les sacrifices nécessaires

pour la défense de notre honneur

aussi longtemps que nous vivrons

(Ibn Hicham, 1967, p. 421).

Dans une des premières expéditions militaires, on constate davantage l'importance de l'honneur tribal désormais défini en termes religieux :

S'ils [les adversaires de Mahomet]

continuent l'idolâtrie et l'errance

La punition de Dieu va être éminente

Nous sommes de purs descendants

de [la tribu] de Ghâlib

D'où la noblesse s'écoule

dans ses branches

(Ibn Hicham, 1967, p. 281-282).

Dans un autre poème, le lien de sang cède la place à la conviction religieuse. Dans le poème suivant, le combattant du djihad (mujahid) menace son frère infidèle :

Le fils de ma mère me reproche d'avoir l'intention de le tuer

Je lui couperai le cou

par une épée pointue

ayant une lame aussi blanche

que le sel par la force d'aiguisement

Mon attaque ne ratera pas son coup

(Ibn Hicham, 1967, p. 369).

Le nouveau zèle religieux est encore mieux exprimé par un autre poète très connu qui est aussi un compagnon de Mahomet, à Médine. Il décrit l'objectif des combattants musulmans comme étant celui de « rechercher le triomphe de la religion de leur Prophète / compter pour rien leur vie et leurs avoirs » (Ibn Hicham, 1967, p. 369).

Entre 613, année de fondation de la citéÉtat de Médine, et 623, l'an du décès de Mahomet, cet esprit poétique guerrier a été alimenté par au moins 115 expéditions militaires et confrontations de toutes sortes (des manœuvres militaires jusqu'à de véritables guerres en passant par des escarmouches; Ibn Hicham, 1991, v. 1). Mahomet lui-même en dirigea 27.

\section{LA GUERRE}

\section{DANS LE DISCOURS CORANIQUE}

Le Coran ainsi que les recueils classiques de hadith sont catégoriques sur la vertu du djihad et sur la valeur du martyre. "L'institution naturelle » de la guerre tribale s'était ainsi transformée en djihad tout en devenant une institution religieuse. Force est de constater que si le Coran ne possède pas de philosophie élaborée de la guerre, il est pourtant catégorique sur son statut et sur ses bases normatives. Les propos coraniques sur la djihad sont dispersés dans plusieurs chapitres (sourates) et sont inclus dans des versets (aya) portant sur d'autres sujets sans lien apparent comme le mariage.

D'abord, on constate une divergence éclatante entre les perspectives de pacifisme absolu et la vision véhiculée par le Coran. Contrairement à la vision qui rejette toute guerre comme un mal, le Coran l'admet en tant que nécessité dans la vie humaine. On y lit en effet que «[...] si Allah ne retenait pas les hommes les uns par les autres, certainement la terre serait corrompue... » (Coran, 2, 252). D'où l'impératif de se préparer à la guerre. "Préparez contre eux (tout) ce que vous pouvez posséder de forces et de groupes de chevaux pour intimider par là les ennemis d'Allah et vos ennemis... »(Coran, 8, 62).

SE SOUMETTRE À UNE CENTRALISATION POLITIQUE QUELCONQUE, QU'ELLE SOIT BASÉE SUR UN CONTRAT SOCIAL OU SUR UN LIEN POLITIQUE TRANS-TRIBAL, DEVIENT INACCEPTABLE.

Selon le Coran, la plus grande exaltation de la guerre vient quand l'acte du djihad dépasse l'humain et devient l'œuvre de Dieu. Allah se place directement à côté de ses soldats et fait la guerre avec eux. «Ce n'est pas vous qui les avez tués; mais c'est Allah qui les a tués. Ce n'est pas toi [s'adressant à Mahomet] qui a lancé (la flèche) [...] mais c'est Allah qui a tiré [...]» (Coran, $8,17)$. Nulle part dans le Coran trouve-t-on de tels éléments narratifs pour définir cette alliance entre Dieu et l'homme.
Vu que le djihad est aussi un devoir de la foi et s'inscrit dans la loi islamique (charia), et que: "Le combat vous a été prescrit mais vous avez de l'aversion pour lui, il se peut que vous haïssiez une chose qui est bonne pour vous... » (Coran, 2, 212-213), on comprend l'importance de l'obligation de la guerre. Le style discursif utilisé dans le verset pour désigner l'obligation de la guerre est le même que celui employé pour les autres actes religieux fondamentaux comme la prière ou le jeûne.

La participation dans la guerre devient ainsi une épreuve divine pour distinguer les vrais croyants des non-croyants (Coran, 3, 186 ; 47, 31 ; 49, 15). Pour le Coran, l'échelle des valeurs privilégie l'obéissance au commandement du djihad (Coran, 4, 95). En lisant les hadith, on se rend compte que le djihad suit immédiatement l'acte de témoignage de foi en Dieu (Nabhani, 1984, p. 20). Le croyant qui combat dans la voie de Dieu avec sa vie et ses avoirs est l'individu le plus sublime (Bukhari, 1974, p. 24 ; Nabhani, 1984, p. 22). Ce combat est même la condition d'admission au paradis. "Le paradis est sous l'ombre des épées » (Nabhani, 1984, p. 27).

Les propos du prophète suivent ainsi de près le texte du Coran. Pour Mahomet, le djihad est une partie fondamentale de sa mission. Il dit dans un hadith très connu que «j'ai donné l'ordre de combattre ces gens [les infidèles] jusqu'à ce qu'ils témoignent qu'il n'y pas de dieux qu'Allah. S'ils le font leur sang et leur propriété sont sauves » (Bukhari, 1974, p. 265 ; Nabhani, 1984, p. 20).

À l'incitation à la guerre s'ajoute la colère d'Allah contre ceux qui ne répondent pas à son appel à la guerre : «[...] à moins que vous ne vous élanciez les premiers (dans le sentier d'Allah) [pour le djihad], il vous punira d'une punition douloureuse...» (Coran, 9, 39). L'importance de l'obligation de la guerre ainsi que des récompenses promises au mujahid (combattant du djihad) est reprise par les juristes dès le deuxième siècle de l'hégire ${ }^{2}$ (voir Sarakhsi, 1989, V. 1, p. 6-28).

\section{LE MARTYRE}

En général, le mot shahada (martyre) a deux sens. D'une part, martyre renvoie au sens très large de celui qui se sacrifie sur la 


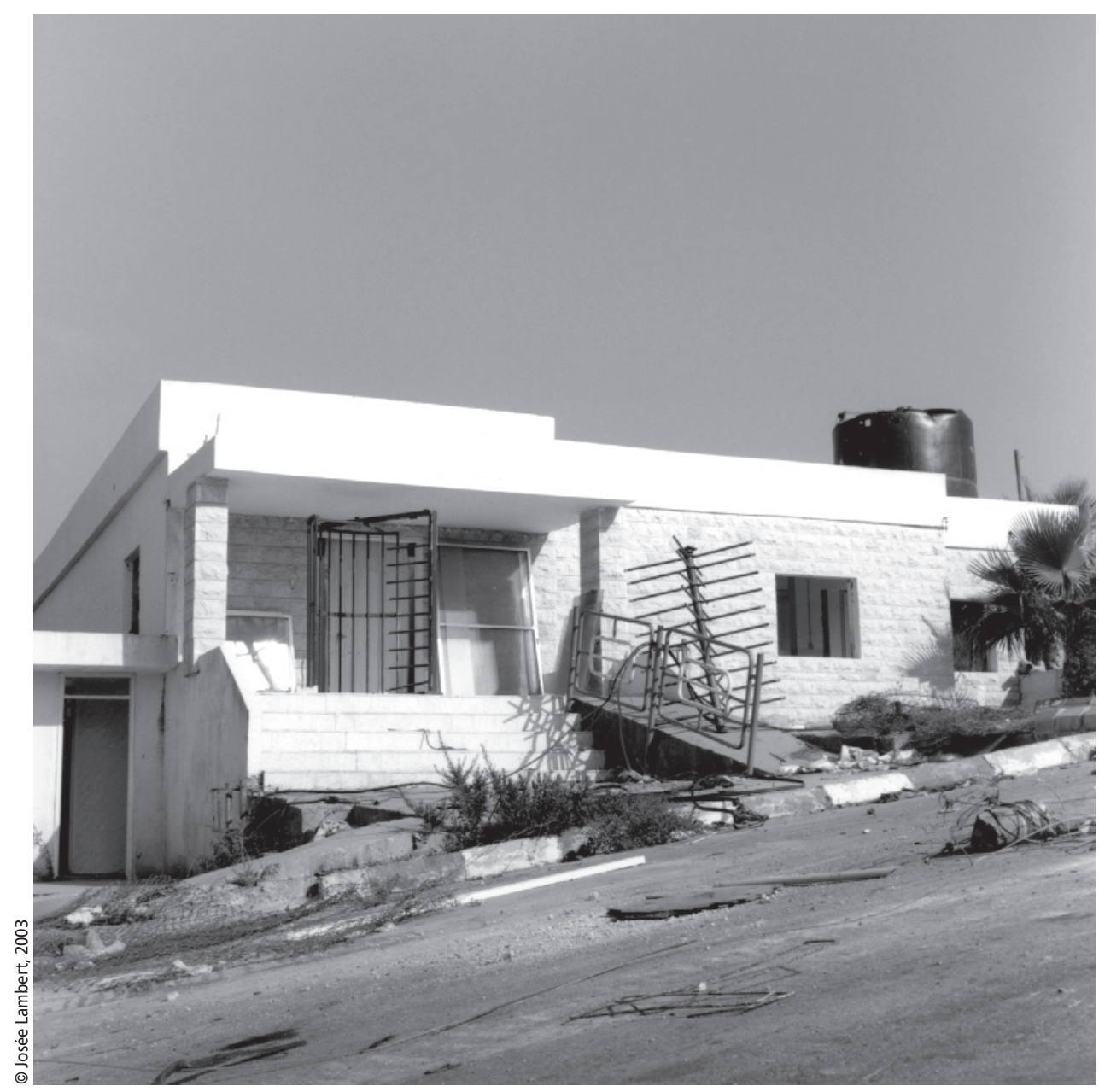

voie de Dieu. Ce sens jaillit de l'intention du croyant et de la nature de ses actes sans que la mort survienne obligatoirement dans un champ de bataille. D'autre part, et c'est le sens qui nous intéresse ici, le martyre signifie la mort du croyant dans une guerre pour la cause de Dieu. Au cours des siècles, un consensus s'est dégagé entre les interprétations et les études coraniques de toutes les confessions sur l'importance privilégiée accordée au martyre par le Coran, mais les deux sens se sont confondus avec comme résultat que sacrifier et mourir pour la cause de Dieu et de l'islam sont devenus tout un.

Le Coran lui accordant une place singulière, le martyre devient le summum de la perfection morale que peut incarner un homme. Il n'est précédé que par le niveau atteint par le prophète lui-même et les récompenses que lui a données Dieu. «Ne dites pas de ceux qui sont tués dans la voie d'Allah : "ce sont des morts". Mais (dites) "ce sont des vivants". Mais vous ne le percevez pas» (Coran, 2, 149). Dans le fameux verset 163 de la sourate 3 , on lit: « Ne comptez pas ceux qui ont été tués dans la voie d'Allah pour des morts. Mais ils sont des vivants auprès de leur Seigneur, pourvus de tout. » Dans les hadiths, il n'y a aucune ambiguité ou équivoque puisqu'on accorde
Ici, le bruit des tampons

de l'administration militaire

assourdit encore les lieux.

jambes, je reste un musulman /j'espère, en échange, gagner une vie proche d'Allah avec les houris [de belles femmes vierges] / habillées comme les plus belles statues avec le paradis suprême consacré à ceux qui y parviennent» (Ibn Hicham, 1967, p. 349).

À cette force psychologique s'ajoute la conviction que les soldats d'Allah sont invincibles. Faire pénétrer dans l'esprit d'un individu la notion de sa propre invincibilité vise à maximiser la force psychologique de ses actes et à écarter tout traumatisme qu'une défaite pourrait infliger dans l'esprit des croyants. Les combattants musulmans pourront ainsi atteindre « [...] une des deux meilleures [choses]. Le martyre pour la cause d'Allah ou la victoire...»(Coran, 9, 52). Et, dans les mots d'un autre mujahid, "Si vous [les infidèles] nous tuez, la vraie foi reste toujours la nôtre / et être tué pour la vérité fera gagner la faveur d'Allah » (Ibn Hicham, 1967, p. 414).

Vertu salvatrice du martyre et invincibilité née de la conviction de l'appui de Dieu se complètent dans un autre principe du Coran, à savoir que la force du nombre ne détermine pas le résultat de la guerre: «Ô toi le prophète! Presse les croyants au combat! S'il y a parmi vous vingt hommes patients, ils l'emportent sur deux cents ; s'il $y$ en a cent parmi vous; ils en vaincront mille...»(Coran, 8, 66). Traduit en langue de mujahid, cela devient: "Leur nombre m'est égal aussi longtemps que je vis, je les combattrai par ma fiable épée» (Ibn Hicham, 1967, p. 316). Le djihad et le shahada en islam classique avaient ainsi une fonction idéologique extrêmement importante. Lacte de sacrifice de soi subordonnait l'égoïsme personnel et la survie physique de l'individu aux priorités de la religion (Klausner, 1987, p. 233).

L'acte du martyre est vu comme un échange spirituel par lequel le musulman offre sa vie pour gagner le paradis. "Ô vous qui croyez! Mettrais-je à votre disposition une marchandise [commerce] pour vous racheter du supplice douloureux ? Croyez en Allah et en son prophète, et combattez avec force dans le sentier d'Allah, avec vos biens et vos personnes. Cela est meilleur pour vous, si vous (le) comprenez » (Coran 61, 10-11). Ce commerce devient plus exaltant, car Allah lui-même rédime le croyant. 
«En vérité, Allah a acheté aux croyants leur personne et leurs biens pour le Paradis qu'ils auront»(Coran 9, 112).

D'ambition mesurée, cet article n'a fait qu'introduire les lecteurs à quelques éléments de base afin de mieux saisir le lien entre les deux concepts de guerre et de martyre. La discussion reste préliminaire, car ces deux éléments ne sont pas isolés et font partie d'un système de valeurs et de pensée islamique.

Nous avons évité les polémiques théologiques entourant ces deux notions en nous limitant à ce qui fait, plus ou moins, consensus entre les théologiens et les islamologues. Certaines questions restent à explorer à partir de ces constats de base. Ces questions deviennent très épineuses, donc loin d'être l'objet de consensus, quand il s'agira de comprendre le martyre dans le contexte des sociétés modernes. Cette relecture de l'islam (ou des islams) reste un des défis les plus ardus des sociétés musulmanes d'aujourd'hui. À notre sens, cette relecture s'oppose à l'islamisme salafiste contemporain (réclamant le retour à l'Islam pur) dans la mesure où elle prône le retour de l'islam à la réalité contemporaine. Cet exercice devient impératif tenant compte de l'effet de la couverture médiatique, pas toujours objective et souvent hâtive, du terrorisme international et des événements du Moyen-Orient. L'association des images et des textes a un impact très important sur la perception parfois chargée d'émotions qu'on retient des musulmans en général. À l'âge de la globalisation de l'information et de l'interdépendance de nos sociétés, le dialogue et la coexistence ne sauraient que renforcer nos ressemblances plutôt qu'exacerber les antagonismes.

\section{Bibliograhie}

ABU DAWUD (1980). Sunan Abi Davud (Propos prophétiques rapportés par Abu Dawud Muhammad Muhi al-Din 'Abd al-Hamid), V. 3, Beyrouth, al-Maktaba Al 'Asryya.

BUKHARI, Ali Mawlana Muhammad (1974). A Manual of Hadith, Préface et traduction par C.E. BOSWORTH, Londres, Curzon Press.

DJAÏT, Hichem (1989). La grande discorde: religion et politique dans l'Islam des origines, Paris, NRF.

DONNER, Fred M. (1991). «The sources of islamic conceptions of war: Just war and jihad: historical and theoretical perspectives on war and peace ", dans John KELSAY et James TURNER JOHNSON (dir.), Western and Islamic Traditions, New York, Greenwood Press, p. 31-71.

GHUNAIMI, Muhammad Talat (1968). The Muslim Conception of International Law and the Western Approach, The Hague, Martins Nijhoff.

HITTI, Philippe K. (1968). Makers of Arab History, New York, St. Martin's Press.

HUNTINGTON, Samuel (2000). Le Choc des civilisations, Paris, Éditions Odile Jacob.

IBN HICHAM, Abd Al-Malik (1967). The life of Muhammad, une traduction de sirat rasul Allah par A. GUILLAUME, Londres, Oxford University Press.

IBN KHALDOUN (1868). Les Prolégomènes, Traduit par SLANE, Paris, Imprimerie nationale.

KLAUSNER, Samuel Z. (1987). "Martyrdom», dans Mircea ELIADE et Charles J. ADAMS et al. (dir.), Encyclopaedia of Religions, New York, Mcmillan.
LAMMENS, Henri, S.I. (1914). Le Berceau de l'Islam: l'Arabie occidentale à la veille de l'hégire. $1^{\text {er }}$ volume (Climat-les Bédouins), Rome, Sumptibus Pontificii Instituti Biblici.

LAWSON, Todd B. (1995). "Martyrdom», dans John L. ESPOSITO, Shahrogh AKHAVI et al. (dir.), The Oxford Encyclopedia of the Modern Islamic World, New York, Oxford University Press.

Le Coran (1929). Traduit en français par Édouard MONTET, Paris, Payot.

MILOT, Jean-René (1993). L'Islam et les musulmans, Québec, Fides.

NABHANI, Yusuf ibn Ismail (1984). AlAhadith al-arba' in fi Fadl al-Jihad wa-alMujahidin (Les Quarante hadiths sur le statut du djihad), Beyrouth, Dar al-Bashair al-Islamiyya.

SARAKHSI (1989). Commentaire du grand livre de Conduite de l'État (Kitab al-Siar alKabir), Traduit par M. HAMIDULLAH. Ankara, Turkiye Diyanet Vakfi.

SHAHID, Irfan (1970). "Pre-islamic Arabia », dans P. M. HOLT, Ann. K. S. LAMBTON et Bernard LEWIS (dir.), The Cambridge History of Islam. v. IA, Cambridge, Cambridge University Press, p. 3-29.

\section{Notes}

1. Ci-après, la traduction des citations des sources en anglais est faite par l'auteur de cet article.

2. Voir ma thèse de doctorat soutenue à l'Université de Montréal: Islam and War: The Disparity between the TechnologicalNormative Evolution of Modern War and the Doctrine of Jihad, chapitre 2. 